\title{
MIMO Using Silicon Patch Antenna
}

\author{
Jagadeesh D ${ }^{\text {a,1 }}$, Karthik E a , Gautam K a , Gopi ${ }^{\text {a }}$ \\ ${ }^{a}$ Department of Electronics and Communication Engineering, \\ Vel Tech High Tech Dr. RR Dr. SR Engineering College, Avadi, Chennai, \\ $T N$, India
}

\begin{abstract}
The growth of mass communication system results the need for the high gain compactable multi-antenna base station by employing the recently introduced Multi-Input Multi-Output (MIMO) concept. A dual-band silicon patch antenna for MIMO operating at $2.4 \mathrm{GHz}$ and $5 \mathrm{GHz}$ has been designed and stimulated results are presented in this paper. The antenna designed with the dielectric constant 2.2 of material RT-duroid results in high efficiency and low substrate loss. ADS software is used for the stimulation of antenna RF-properties and other parameters. With the use of online calculator, the antenna has been designed for $33 \mathrm{~mm}$ of length and $35 \mathrm{~mm}$ of width.
\end{abstract}

Keywords. MIMO, ADS Software, silicon, Microstrip patch antenna.

\section{Introduction}

Multi-Input Multi-Output is a radio antenna communication technology uses multi antennas to receive and transmit data. Wi-Fi, LET, radio and other RF technologies are evolving MIMO concepts [8]. It provides increased link capacity and spectral efficiency. This system provides the advantages of time diversity, frequency diversity and space diversity [1]. It works under the phenomenon of multipath, i.e., the same signal is transmitter at different path through antenna and different frequency [2].

This system has divided into several types based on the number of antenna: SISO (Single Input Single Output) is the simple method which works with single rectenna. It has no diversity and other process. The SIMO (Single Input Multi Output) uses single antenna to transmit data and multiple antennas to receive it. The MISO (Multi Input Single Output) has multiple antenna to transmit data and single antenna to receive data. And the MIMO has multiple antenna to transmit and receive data.

\section{Patch Antenna}

Patch antenna also known as microstrip antenna, which can be printed on circuit board itself [3]. They are at low cost, low profile, lighter in weight and ease to fabricate. This antenna has two layers of fabrication, one for transmission and another for ground. The antenna is measured by length $(l)$ and width (W) of the patch and thickness (h) of the substrate with permittivity $\left(\in_{r}\right)$. The height of the substrate must be smaller than the operating wavelength. The frequency of the antenna is determined the length of the

\footnotetext{
1 Jagadeesh D, Department of Electronics and Communication Engineering, Vel Tech High Tech Dr. RR Dr. SR Engineering College, Avadi, Chennai, TN, India, Email: jagadeeshdamodaran@gmail.com.
} 
patch through the formula $f_{c}=\frac{1}{2 L \sqrt{\epsilon_{0} \epsilon_{r} \mu_{0}}}$. The input impedance is controlled by the width of the patch.

\section{Silicon}

Silicon (Si) is a nonmetallic carbon family element. It is available at $27.7 \%$ of earth atmosphere, which is second most abundant [4]. It has the atomic number of 14, melting point of $1,410^{\circ} \mathrm{C}$ and density of $2.33 \mathrm{~g} / \mathrm{cm}^{2}$. Pure silicon is more reactive to the atmosphere, it's found as common in all rocks as silica (silicon di-oxide) with mixture of oxygen. It has only 0 and +4 oxidation state. It is mainly used as a semiconductor. By the property of conduction silicon is divided into two types. N-type silicon, it's chemically doped with phosphorous for the conduction. It has four electrons with our outer electron bond tightly together. When voltage applied single electron of phosphorous moves and makes negative charge. P-type silicon, it's doped with boron gas for receiving electron.

\section{ADS}

Advance Design System software, It's an electronic design software by Path Wave Design on Keysight Technology [5]. It has a platform to stimulate computer-oriented report of radiofrequency with appropriate antenna [6-7]. The software allows to draw the layout of the antenna and the number of layer or coating with substrate. The software generates the graph with respect to frequency of the antenna working in Figure 3 to Figure 6 . The software allows the dimensional analysis of the proposed system.

\section{Antenna design}

The patch antenna was sketched for $33 * 35 \mathrm{~mm}^{2}$ with the substrate of dielectric constant 2.2 at the thickness of $1.6 \mathrm{~mm}$. The patch and the ground are fabricated with silicon in Figure 1.

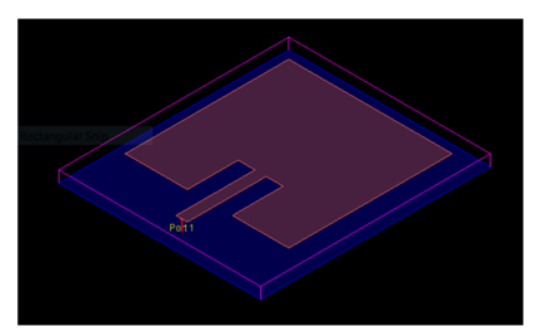

Figure 1. 2D view of antenna

RT-duroid 5880 material is used for substrate, which has good thermal and electric properties. 


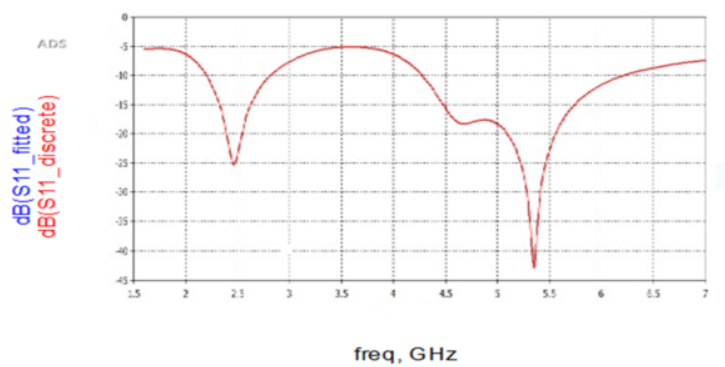

Figure 2. S parameter

The characteristics of the patch antenna designed have been stimulated by ADS software. Figure 2 show the graph between frequency and $S_{11}$.

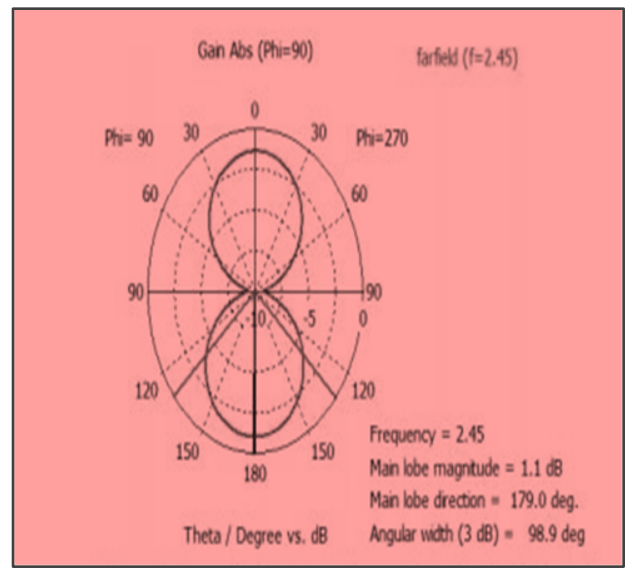

Figure 3. 2D radiation pattern in E-plane for the frequency of $2.4 \mathrm{GHz}$

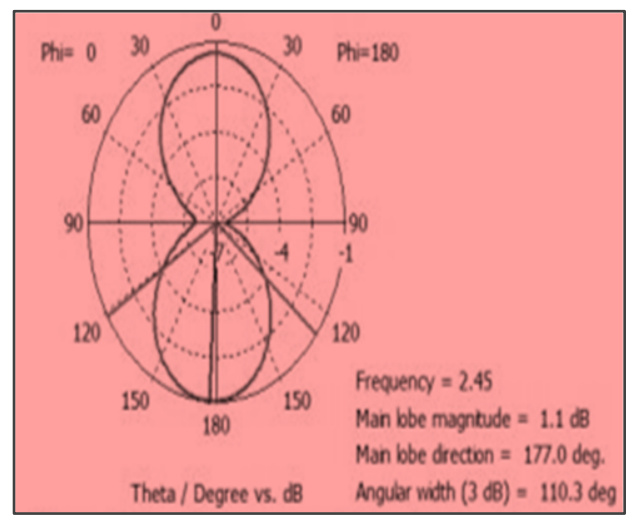

Figure 4. 2D radiation pattern in $\mathrm{H}$-plane for the frequency of $2.4 \mathrm{GHz}$. 


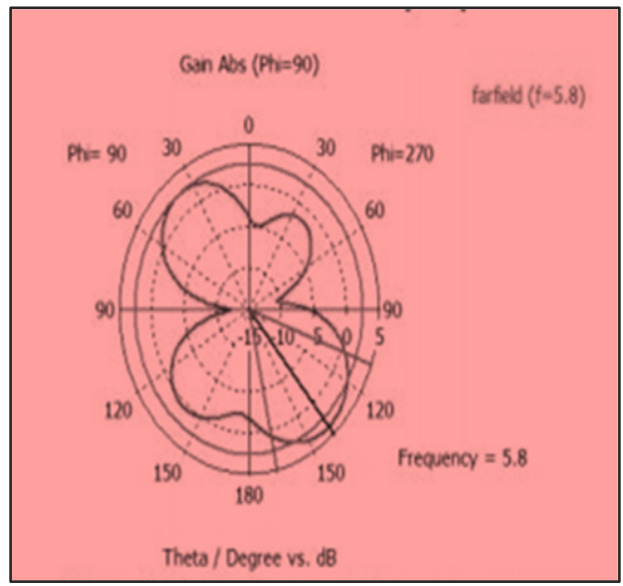

Figure 5. 2D radiation pattern in E-plane for the frequency $5.8 \mathrm{GHz}$.

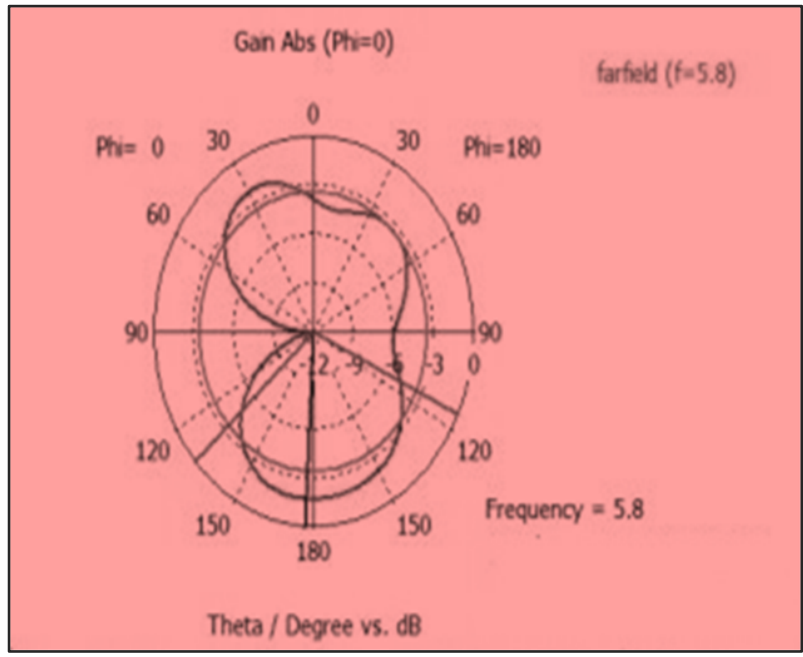

Figure 6. 2D radiation pattern of H-plane for frequency of $5.8 \mathrm{GHz}$.

\section{References}

[1] Rangan, S., Rappaport, T. S., \& Erkip, E. (2014). Millimeter-wave cellular wireless networks: Potentials and challenges. Proceedings of the IEEE, 102(3), 366-385. https://doi.org/10.1109/ JPROC.2014.2299397

[2] Chanclou, P., Cui, A., Geilhardt, F., Nakamura, H., \& Nesset, D. (2012). Network operator requirements for the next generation of optical access networks. IEEE Network, 26(2), 8-14. https://doi.org/10.1109/MNET.2012.6172269

[3] Fontaine, N. K., Doerr, C. R., Mestre, M. A., Ryf, R. R., Winzer, P. J., Buhl, L. L., ... Lingle, R. (2012). Space-division multiplexing and all-optical MIMO demultiplexing using a photonic integrated circuit. In National Fiber Optic Engineers Conference, NFOEC 2012. Optical Society of America (OSA). https://doi.org/10.1364/nfoec.2012.pdp5b.1

[4] C Wiesel, A., Mestre, X., Pages, A., \& Fonollosa, J. R. (2004). Efficient implementation of sphere demodulation. In IEEE Workshop on Signal Processing Advances in Wireless Communications, $S P A W C$ (Vol. 2004-January, pp. 36-40). Institute of Electrical and Electronics Engineers Inc. https://doi.org/10.1109/SPAWC.2003.1318917 
[5] Winzer, P. J. (2014). Making spatial multiplexing a reality. Nature Photonics. Nature Publishing Group. https://doi.org/10.1038/nphoton.2014.58

[6] Miller, D. A. B. (2013). Self-configuring universal linear optical component [Invited]. Photonics Research, 1(1), 1. https://doi.org/10.1364/prj.1.000001

[7] Markkandan, S., Malarvizhi, C., Raja, L., Kalloor, J., Karthi, J., \& Atla, R. (2021), "Highly compact sized circular microstrip patch antenna with partial ground for biomedical applications", Materials Today: Proceedings. https://doi.org/10.1016/j.matpr.2021.04.480

[8] Markkandan, S., Logeshwaran, R., \& Venkateswaran, N. (2021). Analysis of Precoder Decomposition Algorithms for MIMO System Design. IETE Journal of Research, 1-8. https://doi.org/10.1080/03772063.2021.1920848 